\title{
Review: nursing care driven by guidelines improves some process measures and patient outcomes
}

\author{
Thomas L, Cullum N, McColl E, et al. Clinical guidelines in nursing, midwifery and other professions allied to medicine. (Cochrane \\ Review, latest version 24 Nov 1998). In: Cochrane Library. Oxford: Update Software.
}

\author{
Thomas LH, McColl E, Cullum N, et al. Effect of clinical guidelines in nursing, midwifery, and the therapies: a systematic review of \\ evaluations. Quality in Health Care 1998 Dec;7:183-91.
}

\section{Question}

For the disciplines of nursing, midwifery, health visiting, chiropody, speech and language therapy, physiotherapy, occupational therapy, dietetics, clinical psychology, pharmacy, and radiography, do dissemination and implementation of clinical practice guidelines improve professional practice, cost effectiveness, and patient outcomes?

\section{Data sources}

Studies were identified using 9 bibliographic databases and the trials register of the Cochrane Effective Practice and Organisation of Care Group. Experts and libraries of professional organisations were contacted, internet sites were searched, bibliographies were scanned, and 1 journal was handsearched.

\section{Study selection}

Randomised controlled trials (RCTs), controlled before and after studies, or interrupted time series designs were selected if the professions of nursing or allied health were studied and if the dissemination or implementation of a clinical practice guideline was evaluated for changes in clinician behaviour or patient outcomes.

\section{Data extraction}

Data were extracted on study design, participants, clinical area, study comparisons, and process and patient outcomes.

\section{Main results}

18 studies (13 RCTs) met the inclusion criteria. Guidelines were targeted at nurses only (12 studies), nurses and physicians (5 studies), dietitians only (1 study), and a multidisciplinary lipid team that included nurses (1 study). 3 RCTs evaluated guideline dissemination or implementation: 1 of a nurse protocol found positive changes in process (offer of vaccines) and 1 found improved catheter practices; and 1 that measured patient outcomes found more patients in the intervention group declining influenza vaccinations $(\mathrm{p}<0.001)$ and pneumococcal vaccinations $(\mathrm{p}<0.001) .4$ RCTs compared a guideline with no guideline: 1 of 2 found some improvements in care process (labour and delivery). All 4 showed improvements in patient outcomes (quality of life and exercise in patients with diabetes, multiple labour and delivery outcomes, decreased hospital readmission after surgery, and improved lipid values). There were 6 RCTs of skills substitution (nurses taking on tasks conventionally done by a physician). In all 6 studies, nurses showed at least equivalent care processes. 5 studies assessed patient outcomes: 1 study showed no difference in management of dysuria, frequency, and vaginal discharge, and 1 showed no difference in hypertension outcomes; 1 study showed greater satisfaction with nursing care, no difference in symptom relief or complications, but more patients returned with back problems within 3 months; 1 study showed improvement in satisfaction in 6 of 9 elements of headache relief; and 1 study showed improved activated partial thromboplastin time. Methodological quality and data were often limited. Only 1 RCT (of dietitians) included a formal economic evaluation and found a cost efficiency in favour of the control group for 1 outcome (glycated haemoglobin), but no difference in blood glucose concentrations.

\section{Conclusion}

Nursing care driven by guidelines may offer improvement in some process measures and patient outcomes.

Source of funding: National Health Service REDD Programme, UK.

For correspondence: Dr L H Thomas, Centre for Health Services Research, University of Newcastle, 21 Claremont Place, Newcastle upon Tyne NE2 4AA, UK. Fax +44 (0)191 2226043.

\section{Commentary}

This review is the first attempt to address the question of whether the use of clinical guidelines by nurses, midwives, and therapists is clinically or cost effective. Thomas $e t$ al adopt a useful and inclusive definition of practice guidelines that includes care pathways and protocols, similar to other published reviews. ${ }^{1}$ They also set high standards for inclusion (eg, studies that assessed clinical behaviour and patient outcomes). Given their high standards, the bulk of the literature on guideline development and implementation is excluded. This excluded material is often site specific, small scale, and favours action research or other methodologies. The quality of the included studies was often limited; some evidence suggests that more rigorous evaluations are now being done. ${ }^{2}$

Thomas et al clearly show that guidelines can be evaluated for clinician behaviour change and patient outcomes. Their review included studies of varying quality, from RCTs to before and after and time series designs. Because of the wide spectrum of designs, patients, and settings, none of the study findings were combined and meta-analysed.

Although we can see trends that nursing care which is driven by guidelines may offer improvement in some process measures and patient outcomes, we still have a long way to go. For example, research is needed to determine which combinations of educational and implementation strategies (eg, educational outreach) are most likely to lead to changes in nurse behaviour.

$$
\begin{aligned}
& \text { Sally Gooch, RN, RHV, MSc } \\
& \text { Nurse Director/ } \\
& \text { Head of Nursing Development } \\
& \text { Northampton Community Healthcare Trust } \\
& \text { and University College } \\
& \text { Northampton, UK } \\
& 1 \text { Grimshaw JM, Russell IT. Lancet 1993; } \\
& \text { 342:1317-22. } \\
& \text { Worrall G, Chaulk P, Freake D. CMAJ 1997;156: } \\
& 1705-12 \text {. }
\end{aligned}
$$

